\title{
The Acetylcholine Index: An Electroencephalographic Marker of Cholinergic Activity in the Living Human Brain Applied to Alzheimer's Disease and Other Dementias
}

\author{
Magnus Johannsson $^{a} \quad J^{\prime}$ Snaedal ${ }^{b}$ Gisli Holmar Johannesson ${ }^{a}$ \\ Thorkell Eli Gudmundsson $^{b}$ Kristinn Johnsen ${ }^{a}$ \\ a MentisCura ehf., and b Memory Clinic, Geriatric Department, National University Hospital, \\ Reykjavík, Iceland
}

\section{Key Words}

Acetylcholine index · Alzheimer's disease · Electroencephalography · Cholinergic activity

\begin{abstract}
Background: The cholinergic hypothesis is well established and has led to the development of pharmacological treatments for Alzheimer's disease (AD). However, there has previously been no physiological means of monitoring cholinergic activity in vivo. Methods: An electroencephalography (EEG)-based acetylcholine (Ach) index reflecting the cholinergic activity in the brain was developed using data from a scopolamine challenge study. The applicability of the Ach index was examined in an elderly population of healthy controls and patients suffering from various causes of cognitive decline. Results: The Ach index showed a strong reduction in the severe stages of $A D$ dementia. A high correlation was demonstrated between the Ach index and cognitive function. The index was reduced in patients with mild cognitive impairment and prodromal $A D$, indicating a decreased cholinergic activity. When considering the distribution of the Ach index in a population of healthy elderly subjects, an age-related threshold was revealed, beyond which there is a general decline in cholinergic activity. Conclusions: The EEG-based Ach index provides, for the first time, a physiological means of monitoring the cholinergic activity in the human brain in vivo. This has great potential for aiding diagnosis and patient stratification as well as for monitoring disease progression and treatment response.

(C) 2014 S. Karger AG, Basel
\end{abstract}

M.J., J.S., G.H.J. and T.E.G. contributed equally to this work.

Kristinn Johnsen
MentisCura ehf.
Laugavegi 176
IS-105 Reykjavík (Iceland)
E-Mail kristinn @ mentiscura.is

KARGER 125\% 
Johannsson et al.: The Ach Index: An Electroencephalographic Marker of Cholinergic Activity in the Living Human Brain Applied to AD and Other Dementias

\section{Introduction}

Alzheimer's disease (AD) is a progressive degenerative disorder affecting the brain and is the most common cause of cognitive impairment in older individuals [1]. Despite great progress in the understanding of the cognitive profile and neural basis of $\mathrm{AD}$, the underlying cause remains unknown [2]. The focus is now shifting to earlier intervention, thus driving the need for suitable tools for patient stratification and the assessment and prediction of treatment response in the preclinical stages of the disease [3].

The cholinergic hypothesis, introduced in the 1970s, was the first theory to explain AD symptomatology, asserting a transmitter-based pathophysiology of the disease [4]. Early findings examining the brains of AD patients, particularly the cerebral cortex, revealed decreased cholinergic enzymes and a reduction of cholinergic neurons in the majority of cases $[5,6]$. Experimental studies on humans as well as primates indicated the role of acetylcholine (Ach) in learning and memory using cholinergic blockers such as scopolamine to induce memory impairments and cholinergic agonists such as physostigmine to reverse the effects [7-9]. Furthermore, a positive correlation was found between the extent of cholinergic deficits and the severity of dementia in $\operatorname{AD}[10,11]$.

Based on the previous success of considering Parkinson's disease as a dopaminergic deficiency disease, AD became also regarded as a cholinergic deficiency disease. This subsequently resulted in the development of cholinergic treatments with acetylcholinesterase inhibitors (AChEIs). Today, there are 3 approved AChEIs (donepezil, rivastigmine and galantamine) for the treatment of mild-to-moderate AD, and all have been reported to have similar effects by inhibiting the breakdown of Ach [12,13]. Overall, clinical trials have demonstrated that these drugs have positive effects on cognition and global functioning [14, 15], but some patients respond much better than others and the reasons for the difference between clinical responders and non-responders remain unclear [16-18].

Conflicting findings on the clinical applicability of AChEIs and difficulties in the confirmation of a decreased cholinergic activity and loss of cholinergic neurons in the earliest stage of the disease, i.e. mild cognitive impairment [MCI; includes both patients who remain stable and those with so-called prodromal AD (pAD), who will progress to the full disease], have challenged the validity of the cholinergic hypothesis [19-21]. Today, most experts believe that the etiology of $\mathrm{AD}$, as of many other degenerative disorders, is based on multiple causal factors rather than a single etiopathological mechanism [22]. According to this view, cognitive deterioration in $\mathrm{AD}$ is the result of a complicated and not fully understood dysfunction of the complex interplay between different neurotransmitter systems rather than isolated deficits within a single system $[13,23]$. Furthermore, the cholinergic system is found to be disrupted in other types of dementia as well as in various affective disorders, such as schizophrenia, depression, delirium and traumatic brain injury [24-29]. The primary aim of the present work was to develop a means of monitoring the cholinergic activity in the brain in vivo using electroencephalography (EEG). Previous studies have demonstrated the relevance of EEG as an indirect measure of cholinergic activity but have fallen short of developing a single outcome measure with clinical utility [30-33]. We developed a quantitative EEG measure, the Ach index, aimed to reflect the level of cholinergic activity in the brain. The Ach index is derived by comparing the EEG before and after administration of scopolamine, a muscarinic cholinergic antagonist, and thereby statistically determining the multivariate EEG factors that are altered by the pharmacological modulation of the cholinergic system [34]. In practice, this is accomplished by the application of statistical pattern recognition (SPR) methods combined with a genetic algorithm [35], resulting in a discriminatory function that forms the Ach index. In order to demonstrate the applicability of the Ach index, the behaviour of the index was comprehensively 
Johannsson et al.: The Ach Index: An Electroencephalographic Marker of Cholinergic Activity in the Living Human Brain Applied to AD and Other Dementias

examined using EEG recordings from both elderly healthy controls and elderly patients suffering from different stages and types of dementia as well as from a range of expected cholinergic deficits [35].

\section{Materials and Methods}

\section{Data}

Data from a large clinical database of EEG recordings in a range of elderly subjects and patients were used for this study. Clinical cases were recruited in the Memory Clinic of the Geriatric Department, National University Hospital, Reykjavík, Iceland. All clinical cases were investigated using a standard protocol including health information from the patients and their relatives, CT or MRI of the brain, and in milder cases neuropsychological evaluation. In some cases, SPECT of the brain and CSF analysis for $\beta$-amyloid and tau were performed, and in some cases of suspected Lewy body dementia, a DAT scan was performed. All records were evaluated independently by 2 trained geriatricians, and in cases of discrepancy, a consensus by 3 geriatricians was made, one of them not otherwise participating in the study. For the clinical diagnoses, the following criteria were used: the NINDS-ADRDA criteria for the diagnosis of Alzheimer's dementia [36], the NINDSAIREN criteria for vascular dementia [37] and the revised consensus criteria of McKeith et al. [38] for Lewy body dementia. The participants in the control group were recruited by advertising in TV and in service centres for senior citizens; however, some of them were relatives of a patient. Each individual in the control group gave information, if relevant, on the history of head injury with loss of consciousness, neurological diseases such as Parkinson's disease and multiple sclerosis, addiction to alcohol or drugs and any major medical condition. Baseline EEG recordings were made in all subjects upon entry to the database. A baseline Mini-Mental State Examination (MMSE) and Digit Symbol Substitution Test (DSST) were administered to all clinical cases and healthy controls. After registration, the subjects were followed up to enable their allocation to the following categories: (1) normal (NRM) = individuals with no cognitive impairment; (2) stable MCI $(\mathrm{sMCI})=$ individuals diagnosed with $\mathrm{MCI}$ who have been stable for more than 2 years; (3) pAD = individuals who progressed from MCI to AD; (4) AD = individuals who have a clinical diagnosis of AD; (5) Lewy body and Parkinson's dementia (L-P) = individuals in our database with either of these diagnoses; these are included as more severe cholinergic deficits are expected in this group than in the AD group [39]; and (6) vascular dementia (VaD), depression (DPR) and frontal lobe dementia (FLD) patients.

\section{Participants}

EEG registrations from a total of 19 individuals ( 9 healthy elderly individuals and $10 \mathrm{sMCI}$ patients) from the database were analysed for developing the Ach index. All participants had taken part in a previous scopolamine challenge study, in which EEG recordings were made before and after the administration of scopolamine. The mean $( \pm \mathrm{SD})$ age of the healthy elderly individuals was $72.2 \pm 5.3$ and the mean MMSE score was $29.1 \pm 0.9$. For the sMCI group, the mean age was $74.3 \pm 3.2$ and the mean MMSE score was $27.7 \pm 2.2$. More detailed information on the two groups and the scopolamine challenge study can be found in our previous work [34].

In order to provide evidence for the clinical and physiological utility of the Ach index, its properties were investigated using EEG registrations from a total of 948 individuals from the database. The individuals belonged to the following groups in the database: NRM, SMCI, pAD, AD and L-P. For the investigation of the correlation of the Ach index with cognitive performance, individuals from the VaD, DPR and FLD group (with a total of 1,054 EEG registrations) were included in the analyses. The number of subjects in each group along with their age and their MMSE and DSST scores can be found in table 1. No patient was excluded from the study if an EEG registration had been successfully performed.

This study was performed according to good clinical practice requirements and approved by the Icelandic National Bioethics Committee (references No. 04-130, 2004010004). All participants signed a written informed consent form prior to participation, and for those diagnosed with dementia, a written informed consent was obtained from their next of kin.

\section{Analytical and Statistical Procedures}

For the development of the Ach index, the first task was to determine which features are modulated as the cholinergic tone varies in the central nervous system (CNS). This was achieved by analyzing EEG 
Table 1. Composition of groups used for the investigation of the Ach index properties

\begin{tabular}{l|l}
\hline Dement Geriatr Cogn Disord 2015;39:132-142 \\
\hline DOI: 10.1159/000367889 & $\begin{array}{l}\text { @ 2014 S. Karger AG, Basel } \\
\text { www.karger.com/dem }\end{array}$ \\
\hline
\end{tabular}

Johannsson et al.: The Ach Index: An Electroencephalographic Marker of Cholinergic Activity in the Living Human Brain Applied to AD and Other Dementias

\begin{tabular}{llll}
\hline Groups & Age, years & MMSE score & DSST score $^{1}$ \\
\hline NRM $(\mathrm{n}=423)$ & $64.9 \pm 9.2$ & $28.8 \pm 1.5$ & $40.8 \pm 12.4$ \\
SMCI $(\mathrm{n}=78)$ & $73.3 \pm 9.8$ & $27.7 \pm 2.5$ & $28.2 \pm 13.9$ \\
pAD $(\mathrm{n}=62)$ & $76.5 \pm 7.0$ & $26.3 \pm 2.3$ & $25.1 \pm 9.4$ \\
AD $(\mathrm{n}=337)$ & $77.4 \pm 7.6$ & $20.9 \pm 5.3$ & $12.1 \pm 9.6$ \\
L-P $(\mathrm{n}=48)$ & $75.2 \pm 7.6$ & $23.9 \pm 4.5$ & $13.6 \pm 9.1$ \\
VaD $(\mathrm{n}=61)$ & $77.4 \pm 7.6$ & $23.2 \pm 4.3$ & $14.6 \pm 11.0$ \\
DPR $(\mathrm{n}=30)$ & $72.6 \pm 9.2$ & $26.1 \pm 5.5$ & $29.6 \pm 10.2$ \\
FLD $(\mathrm{n}=15)$ & $71.8 \pm 9.6$ & $25.6 \pm 4.8$ & $19.5 \pm 13.7$ \\
\hline
\end{tabular}

Values are presented as means \pm SD.

${ }^{1}$ Average DSST scores were calculated for all subjects completing the test: $342 / 423$ in NRM, 44/78 in sMCI, 32/62 in pAD, 230/337 in AD, 10/48 in L-P, 25/61 in VaD, 18/30 in DPR and 8/15 in FLD.

recordings from a previous scopolamine challenge study, in which EEG recordings were made before and after the subcutaneous administration of $5 \mathrm{mg}$ of scopolamine [34]. First, a classical analysis was undertaken to derive a full set of EEG features (absolute and relative powers at each electrode and a range of coherences between electrode pairs). SPR methods in combination with genetic algorithms were then applied, comparing the subjects with and without the influence of scopolamine to select the relevant features and the combinations that correlated with the scopolamine effects. A more detailed description of the EEG features, SPR methods and genetic algorithm can be found in our previous work [35]. The outcome of this analysis was a series of indices, each using 20 distinct EEG features. All indices had similar statistical properties due to the strong level of correlation between many of the features and had arbitrary scaling. The candidate with the strongest discriminant power based on SPR methods and a genetic algorithm was chosen as the Ach index and is described in figure 1, along with the relevant EEG features that contribute to it. The chosen Ach index had a span of 0-200 without applying any additional scaling factors.

In order to provide evidence for the clinical and physiological utility of the Ach index, its properties were investigated within different groups in the clinical database. Variables are expressed as means \pm SD. Differences between group means were tested for statistical significance using one-way ANOVA. Values of $\mathrm{p}<0.05$ were considered significant. The Bonferroni correction was used to correct for multiple comparisons. The Pearson product-moment correlation coefficient was calculated between selected variables when relevant (e.g. Ach index and cognition). Subsequent analysis was done in the Matlab environment from MathWorks ${ }^{\circledR}$.

\section{Results}

\section{Correlation with Pharmacologically Induced Cholinergic Deficits}

The application of the Ach index to EEG recordings in 9 healthy elderly subjects and 10 sMCI patients from our previously described scopolamine challenge study revealed the expected significant difference before and after the injection of scopolamine. The index decreased in all subjects by a mean of $-31.1 \pm 21.0$ after the injection of scopolamine. In subjects with a high score at baseline, the index decreased more than in subjects with a low score $(r=-0.82, p<0.001)$. Before the injection of scopolamine, the group mean score was $113.7 \pm 21.5$ on the Ach index, as opposed to $82.6 \pm 12.6$ after the injection of scopolamine.

\section{Correlation with Disease-Induced Cholinergic Deficits}

A one-way between-group ANOVA was conducted, followed by a Bonferroni correction post hoc analysis to explore the distributions of the Ach index for different groups from the database. There was a statistically significant difference at the $p<0.05$ level in the Ach index 
Dementia

and Geriatric

Cognitive Disorders

Fig. 1. The EEG features used in the chosen Ach index. Arrows represent coherences and stars represent EEG powers. Red indicates low frequencies $\left(\delta, \theta\right.$ and $\alpha_{1}$ bands), while blue indicates higher frequencies $\left(\alpha_{2}, \beta_{1}, \beta_{2}\right.$ and $\gamma$ bands). Fp2 $-\theta$, Fp $2-\beta_{2}, 02-\gamma$, F8 $-\theta$, T5 $-\gamma$, T5 $-\beta_{1}, \mathrm{Pz}-\gamma, \mathrm{F} 4 /$ $02-\beta_{2}, \mathrm{C} 3 / \mathrm{C} 4-\beta_{1}, \mathrm{C} 3 / \mathrm{C} 4-\beta_{2}$, Fp1/F7 - $\beta_{2}$, Fp2/F8 - $\beta_{2}$, Fp1/ F3 $-\theta$, Fp2/F4 $-\delta$, T6/02 - $\delta$, T6/ $\mathrm{O} 2-\theta, \mathrm{C} 3 / \mathrm{T} 5-\beta_{1}, \mathrm{P} 3 / \mathrm{C} 3-\alpha_{1}, \mathrm{P} 4 /$ C $4-\alpha_{1}$, P4/T6 $-\gamma$. Colors refer to the online version only.

Fig. 2. Box plots of the Ach index for various groups from the database. A significant difference $[F(4,729)=117, p=0.001]$ was found between all pairs of groups using ANOVA with the Bonferroni correction post hoc test.

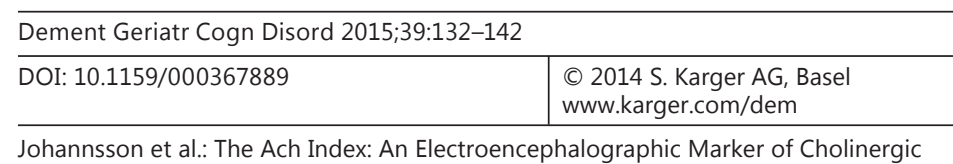

Johannsson et al.: The Ach Index: An Electroencephalographic Marker of Cholinergic
Activity in the Living Human Brain Applied to AD and Other Dementias
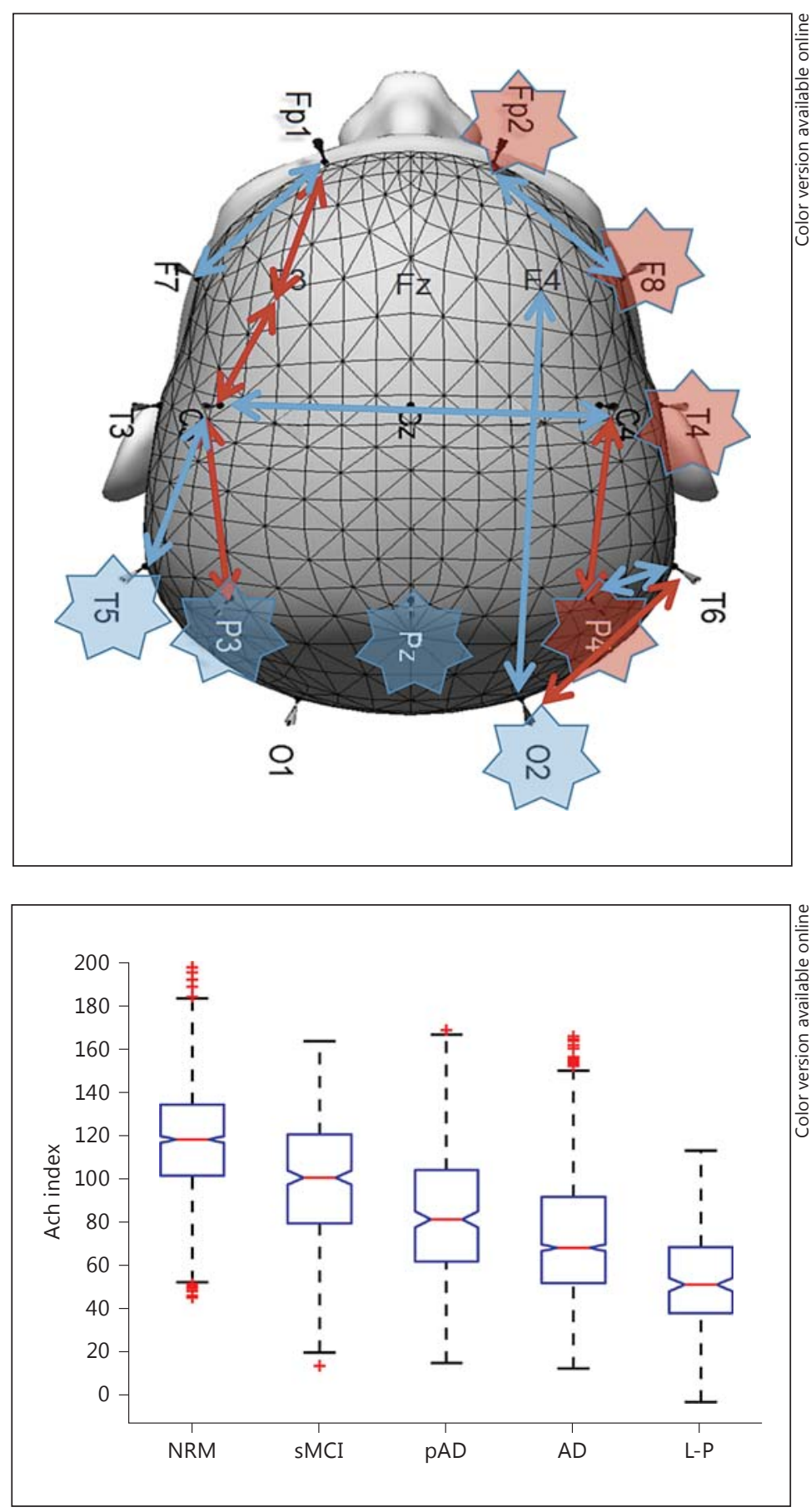

between all pairs of groups $[F(4,729)=117, p=0.001]$ (fig. 2). The means \pm SD of the Ach index for each group are given in table 2 .

\section{Correlation with Cognitive Performance}

Figure 3 shows the relationship between the Ach index and MMSE or DSST scores for all subjects in the database who completed the tests ( $n=1,054$ for MMSE and $n=755$ for DSST) regardless of the clinical group. A strong positive correlation could be observed between the 
Dementia

and Geriatric

Cognitive Disorders

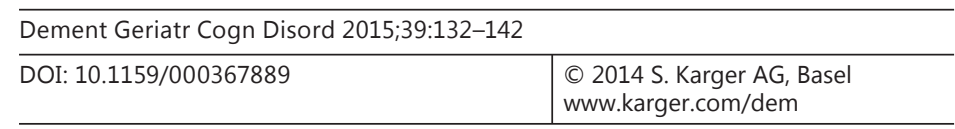

Johannsson et al.: The Ach Index: An Electroencephalographic Marker of Cholinergic Activity in the Living Human Brain Applied to AD and Other Dementias

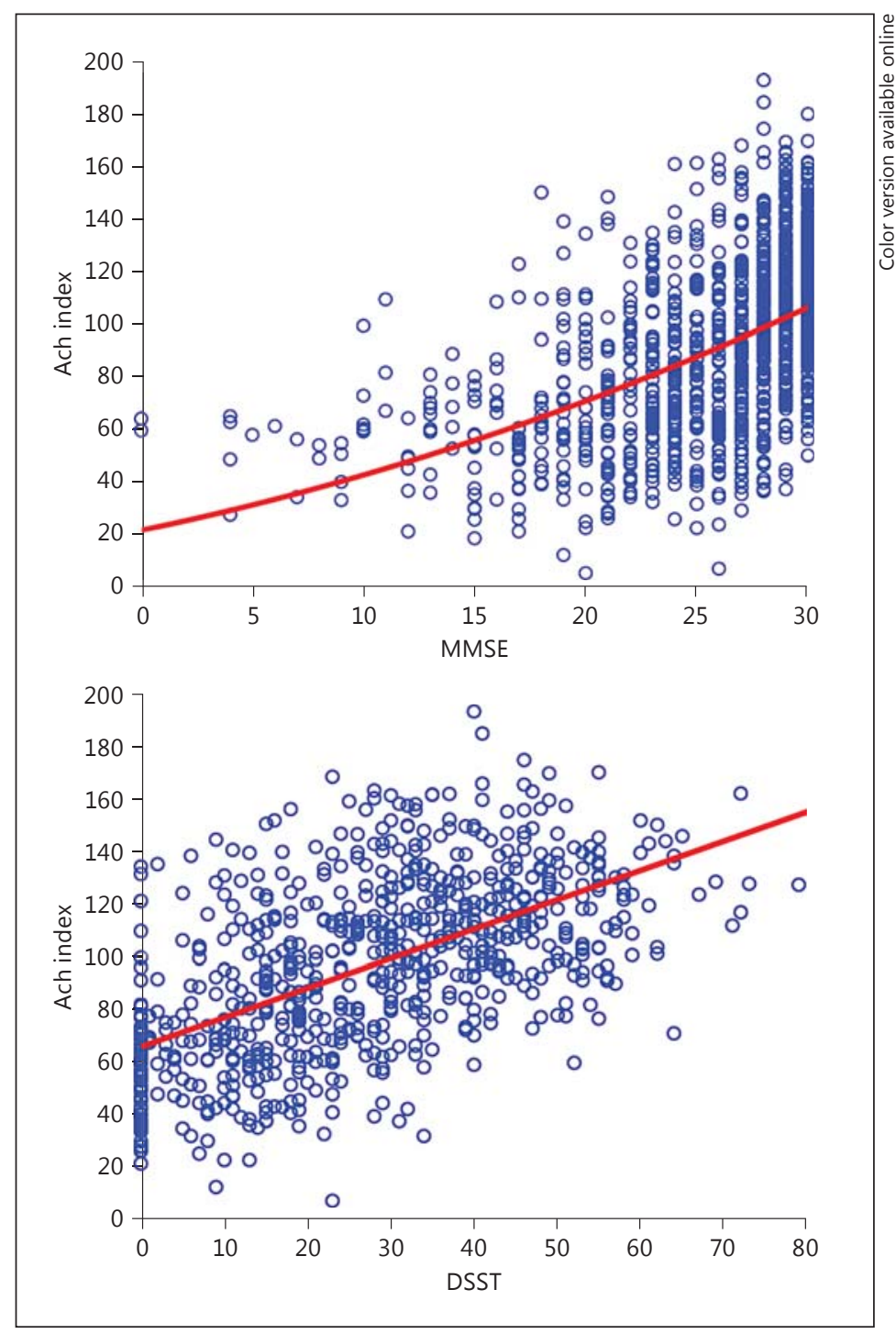

\begin{tabular}{lr}
\hline Groups & Ach index \\
\hline NRM & $118 \pm 24.6$ \\
SMCI & $99 \pm 30.6$ \\
pAD & $83 \pm 28.1$ \\
AD & $73 \pm 30.1$ \\
L-P & $54 \pm 22.6$ \\
\hline
\end{tabular}

Fig. 3. Relationship between the Ach index and MMSE ( $\mathrm{n}=1,054)$ and DSST $(n=755)$ scores for all subjects in the database who completed the test regardless of the clinical group.

Table 2. Mean Ach index $( \pm \mathrm{SD})$ for various groups in the database 
Fig. 4. Relationship between the Ach index and age in healthy subjects $(\mathrm{n}=423)$ with MMSE scores $>27$ and no objective or subjective evidence of cognitive/memory impairment.
Johannsson et al.: The Ach Index: An Electroencephalographic Marker of Cholinergic Activity in the Living Human Brain Applied to AD and Other Dementias

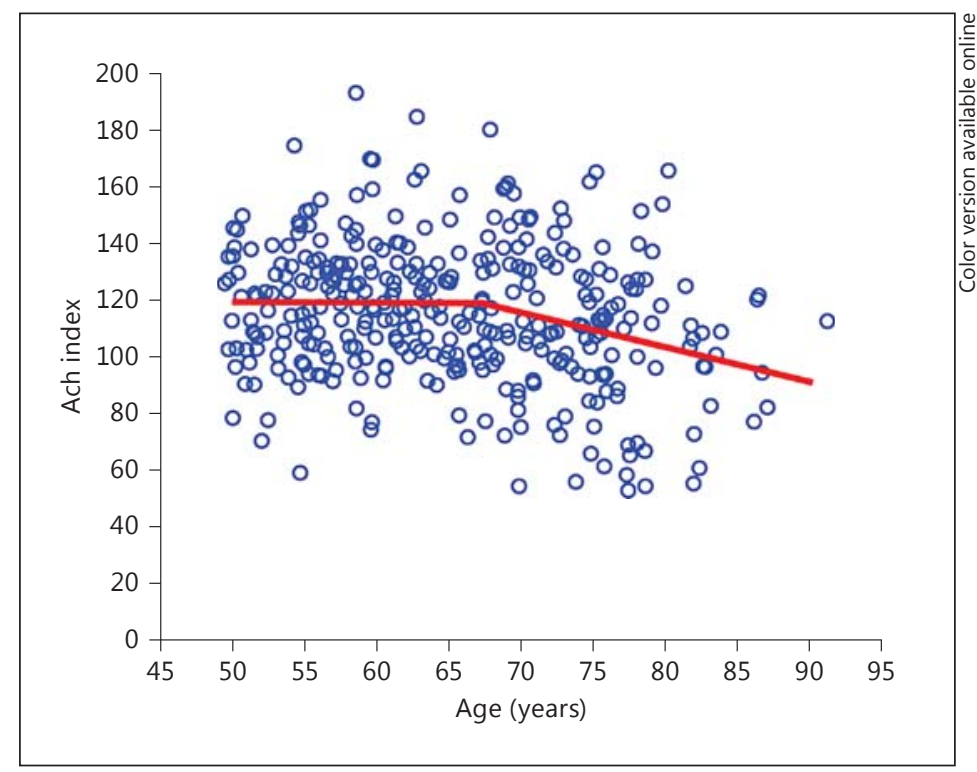

score $>27$ ) in the database. From the age of 48-67 years, there is no correlation evident between the Ach index and age. Between the age of 68 and 93 years, there is a marked deterioration, resulting in a significant negative correlation $(r=-0.24, p<0.003)$.

\section{Discussion}

In the past years, the interest in the cholinergic hypothesis as one of the most important pathophysiological factors of AD has returned. Recent findings in humans as well as animals have supported this claim, and to date, AChEIs remain the only treatment option for mild AD [40]. There is, however, a need for a specific marker of the cholinergic activity in the human brain, especially as the focus shifts to earlier intervention. An effective cholinergic marker would not only assist in the differential diagnosis of dementia but would also enable prediction and assessment of treatment response even in early disease stages. It would be a useful outcome marker in drug development.

In our previous work, we demonstrated the sensitivity of EEG in diagnosing dementia and discriminating between different types by means of a binary classification approach [35]. This paper extends our work by presenting the Ach index as a marker of cholinergic function and by testing its applicability from a clinical as well as a physiological perspective.

The Ach index was generated using training data based on a pharmacological challenge (scopolamine) to modulate the cholinergic tone, which was confirmed by a subsequent re-application of the index to additional data sets. This is important as it ensures that the combination of EEG features chosen is related to the cholinergic system and that the index is not a general marker of, for example, cognitive dysfunction.

Overall, a negative correlation was found between the Ach index and AD disease severity. Significant differences were found between all pairs of groups. The L-P group had a significantly lower Ach index than the AD group, which is in agreement with the expected greater cholinergic impairment [39].

The validity of the cholinergic hypothesis has previously been challenged due to findings failing to confirm decreased cholinergic enzymes and loss of cholinergic neurons in MCI and 
Johannsson et al.: The Ach Index: An Electroencephalographic Marker of Cholinergic Activity in the Living Human Brain Applied to AD and Other Dementias

mild AD in the human brain [19-21]. However, contrary to these findings, the Ach index results reported in this study show a significant deficit in these groups compared to controls (fig. 2). This is in line with recent findings from animal studies and stresses the importance of an in vivo marker of the cholinergic activity in living humans. Most of the previous studies on cholinergic activity in MCI and mild AD have been conducted on postmortem brains focusing mainly on the reduction of 2 enzymes: the choline acetyltransferase and acetylcholinesterase enzymes [12]. Other cholinergic abnormalities such as altered choline transport, release of Ach and nicotinic/muscarinic receptor expression have been suggested to occur in cholinergic neurons at an earlier stage of dysfunction than the reduction of these 2 enzymes. These abnormalities are better reflected in the living brain than in the postmortem brain and may account for previous failures to confirm a decreased cholinergic activity in MCI and mild $\mathrm{AD}[12,13]$. Our findings using the Ach index support this claim.

The cholinergic system has primarily been linked to attention, learning and memory [41]. Two cognitive measures from the database were correlated to the Ach index. These cognitive measures were the MMSE, which assesses general cognition including memory, and the DSST, which assesses executive function and is primarily related to attention. Both measures correlate significantly with the Ach index (fig. 3), which is hence in accordance with the previously established link [41]. Nevertheless, the MMSE and DSST are relatively general measures of cognitive function, and therefore, further research is needed to determine whether the Ach index correlates with more focused and domain-specific measures of cognitive function, such as memory, attention and executive function. In addition, the exploration of the association between cholinergic function and neuropsychiatric and behavioural abnormalities which might have a cholinergic basis (e.g. visual hallucinations) might serve as an interesting field of research [12].

Aging is the strongest risk factor for dementia, and the general decline in cognitive function with age, even in the healthy population, is a confounding factor in dementia research, particularly as most indirect markers of cholinergic activity such as postmortem autopsied tissue analysis do not take these age-related differences into account [22]. Therefore, it is important to understand how the cholinergic system is affected in healthy aging, as reflected by the EEG recordings and the Ach index. Using healthy elderly individuals from the database, the correlation of the Ach index with age was analysed. No correlation was found between the Ach index and age for the subcohort of individuals under the age of 67 years. After the age of 67 years, the deterioration in cholinergic activity was however significant (fig. 4).

These findings indicate an age-related threshold of the activity of the cholinergic system after which it deteriorates, which could explain the increased vulnerability of the elderly to succumb to cognitive decline and dementia. This hypothesis is in line with results from animal studies in which aged brain neurons in the basal forebrain have been shown to function normally until stressed [42]. According to this view, insults to the cholinergic neurons affect their ability to produce sufficient levels of transmitter release to maintain normal functioning [12]. In the human brain, such insults to the cholinergic system can be caused by multiple factors over the life span [40]. The correlation between the Ach index and age suggests that, up to a certain point, the cholinergic system can compensate for these insults and keep the overall function of the system within normal levels. However, as the cholinergic neurons get older, previous or new insults or stress factors such as stroke, anaesthesia, circadian disruption or amyloid plaques may induce cholinergic deficits severe enough to result in clinical symptoms such as memory problems. Our work in a population of healthy elderly individuals suggests that this regime shift takes place, on average, at around 67 years of age, which fits approximately to the average onset of clinical symptoms in dementia, although of course this varies between the different types of dementia and is dependent on the underlying pathology. The greatly increased susceptibility in the oldest 
Johannsson et al.: The Ach Index: An Electroencephalographic Marker of Cholinergic Activity in the Living Human Brain Applied to AD and Other Dementias

group in the elderly population might even account for cases of dementia in which no underlying pathology is evident [43].

Interestingly, there is a rather large variability of the Ach index within the healthy elderly subjects as well as in the clinical groups, suggesting that an overlap exists and that age is not the only factor affecting the index (fig. 2, 4; table 2). This is most likely due to factors such as the general state of the individuals during recording (sleepiness and stress), anticholinergic drugs or previous traumas to the brain known to affect the cholinergic system [12].

Finally, we need to remember that different neurotransmitter systems are affected in different dementias. As all neurotransmitter systems are interconnected, a decrease of function in one system affects the function of the others, reflecting an up- and downstream causal chain $[13,23]$. Hence, as the interconnectivity of different neurotransmitter systems is not fully understood, an index reflecting only one such system cannot provide the complete picture. However, the approach described herein can be applied more generally, and future research might result in the development of EEG-based indexes for other neurotransmitter systems.

\section{Conclusions}

This study introduces a novel approach to monitor the cholinergic activity in the human brain and presents initial evidence for its clinical and physiological utility. In particular, the results in MCI and mild AD patients agree with previous findings from animal studies that have previously been difficult to repeat in humans. This highlights the importance of a functional in vivo marker of the cholinergic activity in the living human brain for the study of dementia and other diseases involving cholinergic derangement.

As a functional marker of cholinergic activity, the Ach index has the capacity to improve the differential diagnosis of dementia and the monitoring of disease progression. In addition, it has great potential for predicting and monitoring treatment response, including discriminating AChEI treatment responders from non-responders, and as an outcome marker in drug development.

\section{Acknowledgements}

This work was funded in part by the Icelandic Technology Development Fund, grants No. 051221006 and 081206008 . We also thank John-Paul Taylor and Fred Wilson for carefully reading the manuscript and providing helpful comments.

\section{Disclosure Statement}

M.J., G.H.J. and K.J. are employees of MentisCura ehf., which is a privately owned for-profit enterprise. J.S. and T.E.G. declare that they have no competing interests.

\section{References}

1 Ferri CP, Prince M, Brayne C, et al: Global prevalence of dementia: a Delphi consensus study. Lancet 2005;366: 2112-2117.

-2 Goedert M, Spillantini MG: A century of Alzheimer's disease. Science 2006;314:777-781.

3 Reiman EM: Alzheimer's disease and other dementias: advances in 2013. Lancet Neurol 2014;13:3-5.

4 Bartus RT, Dean RL, Beer B, Lippa AS: The cholinergic hypothesis of geriatric memory dysfunction. Science 1982;217:408-414. 
Johannsson et al.: The Ach Index: An Electroencephalographic Marker of Cholinergic Activity in the Living Human Brain Applied to AD and Other Dementias

5 Bowen DM, Smith CB, White P, Davison AN: Neurotransmitter-related enzymes and indices of hypoxia in senile dementia and other abiotrophies. Brain J Neurol 1976;99:459-496.

6 Davies P, Maloney AJ: Selective loss of central cholinergic neurons in Alzheimer's disease. Lancet 1976;2:1403.

7 Bartus RT: Evidence for a direct cholinergic involvement in the scopolamine-induced amnesia in monkeys: effects of concurrent administration of physostigmine and methylphenidate with scopolamine. Pharmacol Biochem Behav 1978;9:833-836.

8 Drachman DA: Memory and cognitive function in man: does the cholinergic system have a specific role? Neurology 1977;27:783-790.

19 Drachman DA, Leavitt J: Human memory and the cholinergic system. A relationship to aging? Arch Neurol 1974;30:113-121.

10 Perry EK, Blessed G, Tomlinson BE, et al: Neurochemical activities in human temporal lobe related to aging and Alzheimer-type changes. Neurobiol Aging 1981;2:251-256.

11 Francis PT, Palmer AM, Sims NR, et al: Neurochemical studies of early-onset Alzheimer's disease. Possible influence on treatment. N Engl J Med 1985;313:7-11.

$\$ 12$ Terry AV, Buccafusco JJ: The cholinergic hypothesis of age and Alzheimer's disease-related cognitive deficits: recent challenges and their implications for novel drug development. J Pharmacol Exp Ther 2003;306:821827.

13 Martorana A, Esposito Z, Koch G: Beyond the cholinergic hypothesis: do current drugs work in Alzheimer's disease? CNS Neurosci Ther 2010;16:235-245.

14 Birks J: Cholinesterase inhibitors for Alzheimer's disease. Cochrane Database Syst Rev 2006;CD005593

15 Kaduszkiewicz H, Zimmermann T, Beck-Bornholdt HP, van den Bussche H: Cholinesterase inhibitors for patients with Alzheimer's disease: systematic review of randomised clinical trials. BMJ 2005;331:321-327.

-16 Courtney C, Farrell D, Gray R, et al: Long-term donepezil treatment in 565 patients with Alzheimer's disease (AD2000): randomised double-blind trial. Lancet 2004;363:2105-2115.

17 Connelly PJ, Prentice NP, Fowler KG: Predicting the outcome of cholinesterase inhibitor treatment in Alzheimer's disease. J Neurol Neurosurg Psychiatry 2005;76:320-324.

18 Frölich L: The cholinergic pathology in Alzheimer's disease - discrepancies between clinical experience and pathophysiological findings. J Neural Transm 2002;109:1003-1013.

19 Davis KL, Mohs RC, Marin D, et al: Cholinergic markers in elderly patients with early signs of Alzheimer disease. JAMA 1999;281:1401-1406.

20 DeKosky ST, Ikonomovic MD; Styren SD, et al: Upregulation of choline acetyltransferase activity in hippocampus and frontal cortex of elderly subjects with mild cognitive impairment. Ann Neurol 2002;51:145-155.

-21 Gilmor ML, Erickson JD; Varogui H, et al: Preservation of nucleus basalis neurons containing choline acetyltransferase and the vesicular acetylcholine transporter in the elderly with mild cognitive impairment and early Alzheimer's disease. J Comp Neurol 1999;411:693-704.

22 Craig LA, Hong NS, McDonald RJ: Revisiting the cholinergic hypothesis in the development of Alzheimer's disease. Neurosci Biobehav Rev 2011;35:1397-1409.

23 Steckler T, Sahgal A: The role of serotonergic-cholinergic interactions in the mediation of cognitive behaviour. Behav Brain Res 1995;67:165-199.

24 Overshott R, Burns A: Cholinesterase inhibitors: in search of cholinergic deficits. Adv Psychiatr Treat 2005; 11:321-324.

25 Arciniegas DB: The cholinergic hypothesis of cognitive impairment caused by traumatic brain injury. Curr Psychiatry Rep 2003;5:391-399.

26 Battaglia M: Beyond the usual suspects: a cholinergic route for panic attacks. Mol Psychiatry 2002;7:239-246.

27 Dilsaver SC, Coffman JA: Cholinergic hypothesis of depression: a reappraisal. J Clin Psychopharmacol 1989;9: 173-179.

28 Hshieh TT, Fong TG, Marcantonio ER, Inouye SK: Cholinergic deficiency hypothesis in delirium: a synthesis of current evidence. J Gerontol A Biol Sci Med Sci 2008;63:764-772.

-29 Raedler TJ, Bymaster FP, Tandon R, Copolov D, Dean B: Towards a muscarinic hypothesis of schizophrenia. Mol Psychiatry 2006;12:232-246.

30 Agnoli A, Martucci N, Manna V, Conti L, Fioravanti M: Effect of cholinergic and anticholinergic drugs on shortterm memory in Alzheimer's dementia: a neuropsychological and computerized electroencephalographic study. Clin Neuropharmacol 1983;6:311-323.

-31 Jeong J: EEG dynamics in patients with Alzheimer's disease. Clin Neurophysiol 2004;115:1490-1505.

-32 Stewart DJ, Macfabe DF, Vanderwolf CH: Cholinergic activation of the electrocorticogram: role of the substantia innominata and effects of atropine and quinuclidinyl benzilate. Brain Res 1984;322:219-232.

33 Babiloni C, Frisoni GB, Vecchio F, et al: Global functional coupling of resting EEG rhythms is related to whitematter lesions along the cholinergic tracts in subjects with amnesic mild cognitive impairment. J Alzheimers Dis 2010;19:859-871.

-34 Snaedal J, Johanesson GH, Gudmundsson TE, et al: The use of EEG in Alzheimer's disease, with and without scopolamine - a pilot study. Clin Neurophysiol 2010;121:836-841.

-35 Snaedal J, Johanesson GH, Gudmundsson TE, et al: Diagnostic accuracy of statistical pattern recognition of electroencephalogram registration in evaluation of cognitive impairment and dementia. Dement Geriatr Cogn Disord 2012;34:51-60. 
Johannsson et al.: The Ach Index: An Electroencephalographic Marker of Cholinergic Activity in the Living Human Brain Applied to AD and Other Dementias

-36 McKhann G, Drachman D, Folstein M, et al: Clinical diagnosis of Alzheimer's disease: report of the NINCDSADRDA Work Group under the auspices of Department of Health and Human Services Task Force on Alzheimer's Disease. Neurology 1984;34:939-944.

-37 Román GC, Tatemichi TK, Erkinjuntti T, et al: Vascular dementia: diagnostic criteria for research studies. Report of the NINDS-AIREN International Workshop. Neurology 1993;43:250-260.

-38 McKeith IG, Dickson DW, Lowe J, et al: Diagnosis and management of dementia with Lewy bodies: third report of the DLB Consortium. Neurology 2005;65:1863-1872.

39 Aarsland D, Mosimann UP, McKeith IG: Role of cholinesterase inhibitors in Parkinson's disease and dementia with Lewy bodies. J Geriatr Psychiatry Neurol 2004;17:164-171.

40 Contestabile A: The history of the cholinergic hypothesis. Behav Brain Res 2011;221:334-340.

41 Mesulam M: The cholinergic lesion of Alzheimer's disease: pivotal factor or side show? Learn Mem 2004;11: 43-49.

42 Burk JA, Herzog CD, Porter MC, Sarter M: Interactions between aging and cortical cholinergic deafferentation on attention. Neurobiol Aging 2002;23:467-477.

43 Haroutunian V, Schnaider-Beeri M, Schmeidler J, et al: Role of the neuropathology of Alzheimer disease in dementia in the oldest-old. Arch Neurol 2008;65:1211-1217. 